\title{
Modèle énergétique uniboite de la croissance des huitres (Crassostrea gigas) dans le bassin de Marennes-Oléron
}

\author{
C. Bacher, M. Héral, J. M. Deslous-Paoli et D. Razet \\ Ifremer, Laboratoire Ecosystèmes Conchylicoles, BP 133, 17390, La Tremblade, France
}

Bacher, C., M. Hérai, 1. M. Deslous-Paoli et D. Razet. 1991. Modèle énergétique uniboite de la croissance des huîtres (Crassostrea gigas) dans le bassin de Marennes-Oléron. Can. J. Fish. Aquat. Sci. 48: 391-404.

Un modèle de croissance des huitres (Crassostrea gigas) dans le bassin de Marennes-Oléron est élaboré afin d'étudier la notion de capacité trophique. Les facteurs environnementaux pris en compte sont le seston total et la somme des équivalents énergétiques des protides, lipides et glucides de la colonne d'eau. Leur évolution temporelle sur 2 ans est reconstituée avec un pas de temps journalier à l'aide d'un modèle régressif fonction du courant de marée, de la hauteur d'eau et de la force du vent. A partir de ces séries, un modèle de bilan énergétique, comprenant un terme d'assimilation et un terme de respiration, est construit pour simuler la croissance d'une huitre pendant 2 ans. Connaissant le nombre d'individus dans le bassin, le débit entrant et le volume moyens des masses d'eau dans le bassin, ce modèle permet d'estimer à $7 \%$ le pourcentage d'énergie utilisée par la population. En faisant varier de façon théorique l'effectif de la population, on montre que la croissance individuelle est sensible au stock d'huitres. Ce calcul introduit la notion de capacité trophique et montre l'intérêt d'une approche plus élaborée de couplage d'un modèle de transport de la nourriture et du modèle de croissance étudié.

The growth of oysters (Crassostrea gigas) is modeled in order to study the trophic capacity of the Bay of MarennesOléron. Total seston and amount of protids, lipids, and carbohydrates converted to energy are the environmental factors taken into account. Time series of these variables are derived during $2 \mathrm{yr}$ with a daily time step from a linear regression including the tidal velocity, the height of the water column, and the wind velocity. A model of the energetic budget based on assimilation and respiration terms and depending on the previous time series is run to simulate the individual growth of an oyster. The knowledge of the number of oysters, the flow of water entering the bay, and the volume of the bay allows us then to estimate that $7 \%$ of the available energy is used by the population. The number of individuals is given theoretical values to show that the impact of the amount of oysters on the growth of an individual is nonnegligible. The trophic capacity of the bay is then defined and it is pointed out that more information may be expected from a model coupling the transport of particulate matter and the growth of oysters.

Reçu le 8 décembre 1988

Accepté le 20 septembre 1990

Received December 8, 1988

(J9973)

Accepted September 20, 1990

L es relations trophiques des bivalves ont le plus souvent été modélisées de façon empirique. Ainsi Bodoy et PlanteCuny (1984), Héral et al. (1984) établissent des régressions linéaires du poids individuel en fonction des paramètres du milieu afin de déterminer les paramètres hydrobiologiques ou sédimentologiques les plus importants. Goulletquer et Bacher (1989) généralisent cette démarche par l'introduction de régressions non linéaires et comparent les performances de croissance en différents sites. Ces modèles empiriques peuvent être prédictifs et généralement applicables à toute étude de relations trophiques dans le même site ou un site de caractéristiques hydrobiologiques voisines. Ils manquent cependant de réalisme (Levins 1966) et ne permettent pas de mesurer la quantité de nourriture retirée du milieu par les filtreurs. Il est cependant notable que la nourriture devient limitante pour un stock élevé (Héral et al 1986a, 1988) et une augmentation de stock induit un ralentissement de croissance.

Ainsi dans le bassin de Marennes-Oléron, important centre de production d'huîtres (Crassostrea gigas), la relation entre le stock d'huîtres et les performances de croissance permet d'expliciter la notion de capacité trophique du bassin, définie comme la capacité d'élevage d'un stock initial d'individus devant être amenés à une taille commerciale déterminée. Les modèles de bilan énergétique permettent d'établir un bilan des besoins et dépenses énergétiques globaux (Rodhouse 1979). Cela implique l'identification de la nourriture potentielle et l'écriture des lois de consommation, d'assimilation et de respiration en fonction des paramètres environnementaux. Ce type de modèle a été appliqué à Mytilus edulis par Bayne et al. (1976), Verhagen (1982) et Harris et al. (1984). On trouve également leur utilisation en aquaculture pour certaines espèces de poissons ( $\mathrm{Maj}$ kowski et Waiwood 1981; Kitchell et Breck 1980).

Cette étude a pour but le calage d'un modèle simple de croissance et d'assimilation de Crassostrea gigas à l'aide de données acquises principalement in situ en 1979 et 1980 . Le problème de la variabilité hydrobiologique des variables explicatives du bilan énergétique en milieu estuarien est pris en compte. Le modèle est appliqué de façon théorique au bassin de MarennesOléron considéré comme un "raceway" de débit connu afin de calculer l'impact potentiel de l'évolution du stock sur la croissance (schéma 1).

\section{Matériels et méthodes}

\section{Plan d'échantillonnage}

Les données hydrobiologiques ont été acquises en un poinı central du bassin (banc Dagnas, fig. 1) par Héral et al. (1983a' 


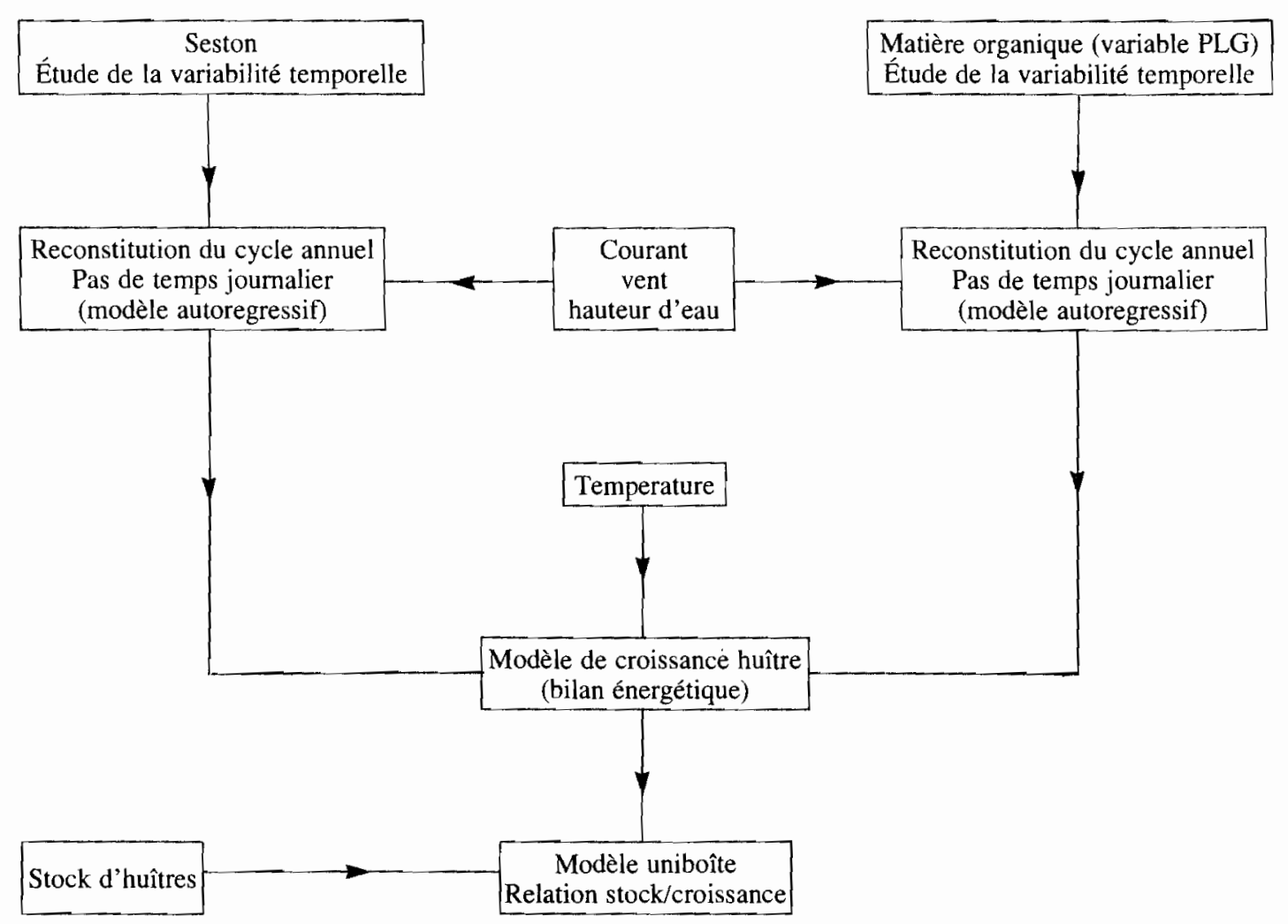

SCHÉMA 1. Organigramme des différents calculs réalisés dans cette étude afin de modéliser la relation entre le stock et les performances de croissance des huîtres Crassostrea gigas dans le bassin de Marennes-Oléron.

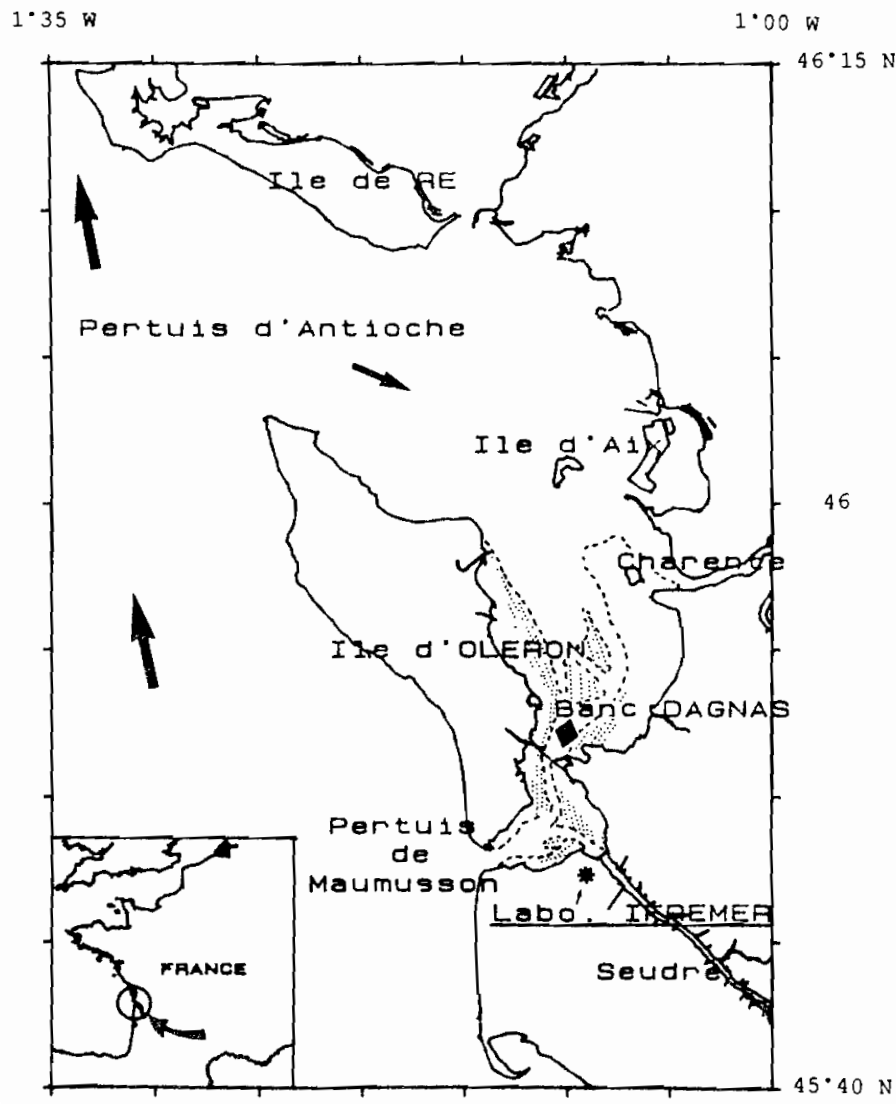

Fig. 1. Carte du bassin de Marennes-Oléron et emplacement du site étudié. et Deslous-Paoli et Héral (1984). Le plan d’échantillonnage a consisté à suivre une population d'huîtres $\left(200\right.$ individus $\left./ \mathrm{m}^{2}\right)$ et les paramètres de la colonne d'eau pendant deux ans, en 1979 et 1980. Des prélèvements mensuels sur 10 individus ont servi à mesurer le poids sec sans cendre, le poids de coquille, les constituants biochimiques et la valeur énergétique de la chair. Les paramètres hydrobiologiques seston organique, seston minéral, protides, lipides, glucides, température, chlorophylle $a$, phéopigments ont été mesurés à deux hauteurs de la colonne d'eau selon un rythme bimensuel, pendant une marée de vive-eau et une marée de morte-eau. A chacune de ces marées six prélèvements espacés de 2 h furent effectués de façon à estimer la variabilité horaire.

La nourriture potentielle est définie comme la somme des équivalents énergétiques des protides, lipides, glucides de la colonne d'eau (Héral 1985). Par la suite cette variable sera appelée PLG.

\section{Variabilité du milieu}

Le bassin de Marennes-Oléron est de type macrotidal. D'après Héral et al. (1987), quatre sources de variabilité expliquent les cycles des traceurs particulaires : gradient vertical, cycle horaire de la marée (période $12: 30$ ), cycle lunaire (période 15 jours) et tendance saisonnière (période 1 an). Une analyse de variance (ANOVA) sur les différentes composantes du seston et de la variable PLG en 1979 et 1980 a permis de hiérarchiser les effets des différentes perturbations d'après les niveaux de signification des trois facteurs date, type de marée (morte-eau ou vive-eau) et hauteur du prélèvement. Le modèle s'écrit

$$
Y_{i j k}=+A_{i}+B_{j}+C_{k}+A B_{i j}+A C_{i k}+B C_{j k}+\epsilon_{i j k}
$$




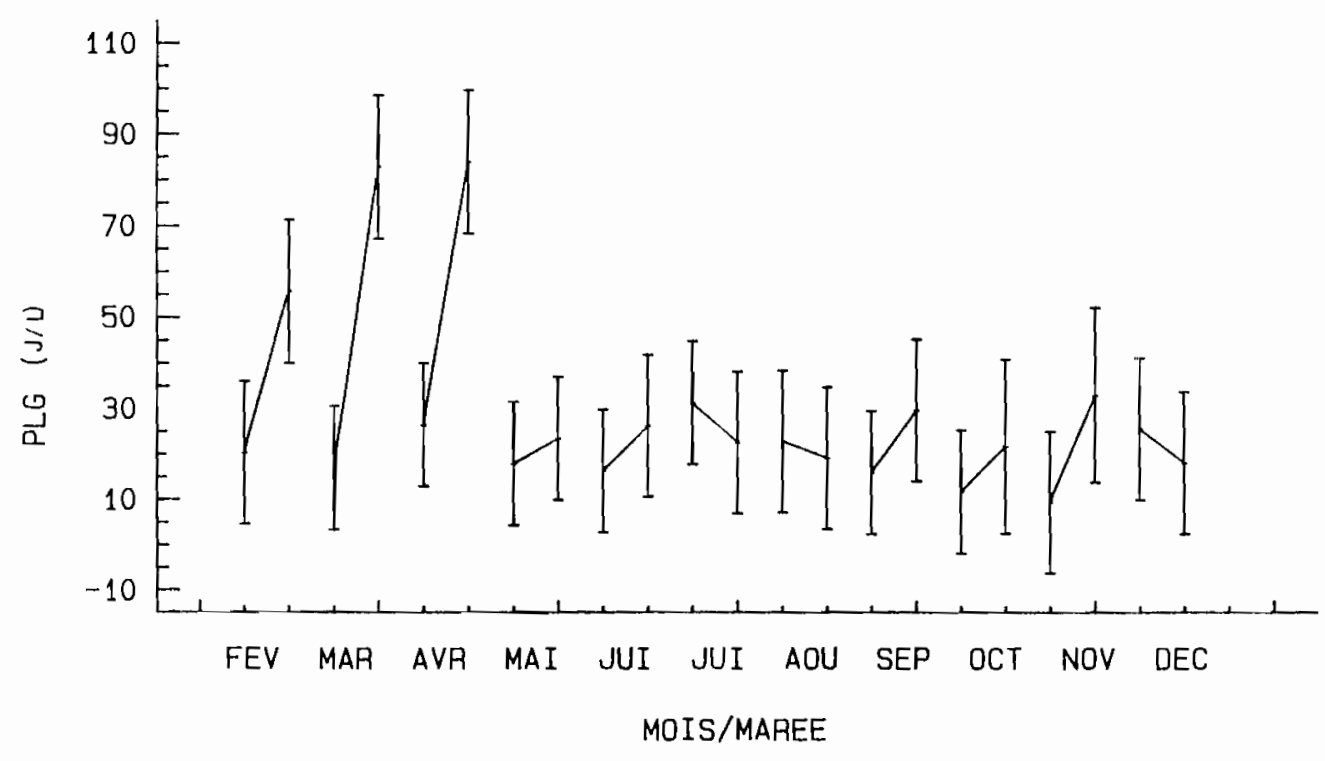

b) INTERACTION ENTRE LES FACTEURS
MOIS ET MAREE EN 1980

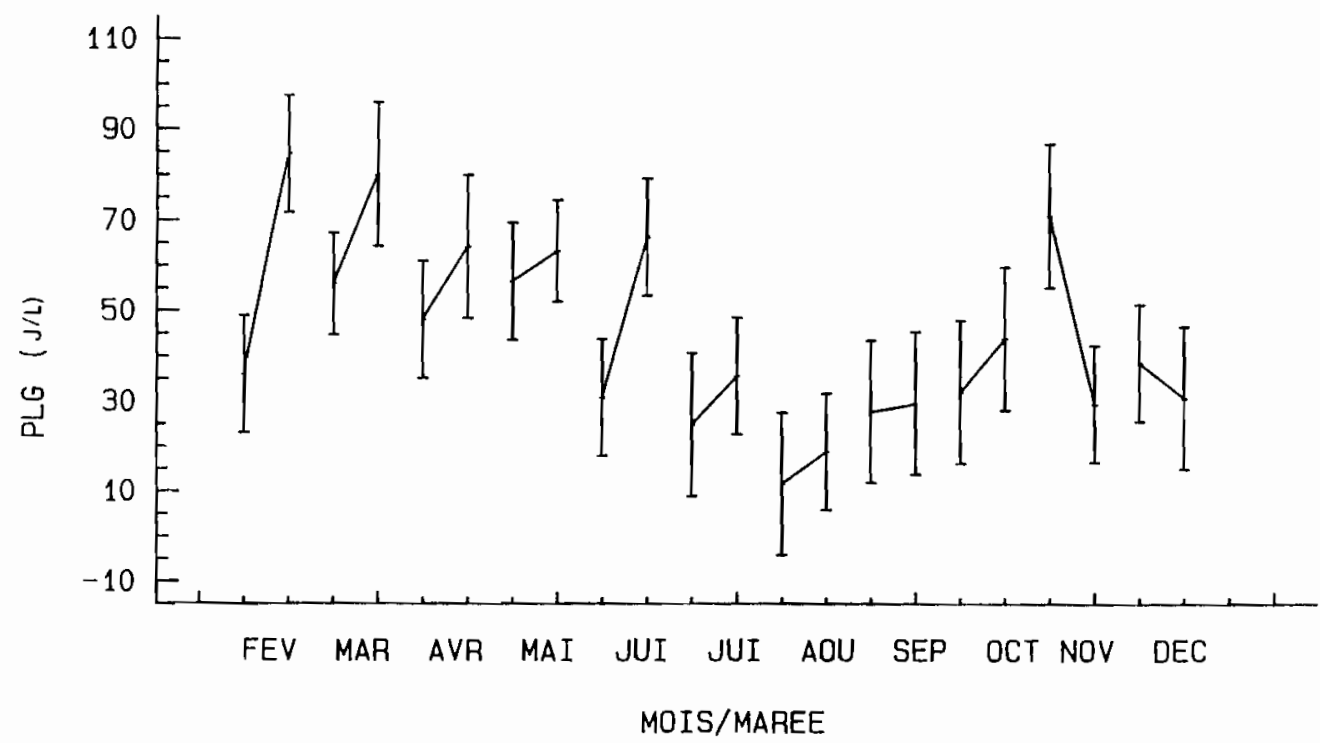

FIG. 2. ANOVA des variables seston et PLG en fonction des facteurs date (mois de février à décembre) et coefficient de marée (mortes-eaux, vives-eaux) en 1979 et 1980. Le graphe met en évidence l'interaction entre les deux facteurs, l'augmentation des concentrations particulaires par marées de viveseaux et l'évolution saisonnière des variables. Pour chaque mois, la première valeur se rapporte à la marée de mortes-eaux, la deuxième à celle de vives-eaux. a) Variable PLG en 1979; b) variable PLG en 1980; c) variable seston en 1979; d) variable seston en 1980.

où $\mathrm{A}_{i}, B_{j}$, et $C_{k}$ désignent les trois facteurs hauteur du prélèvement $\left(A_{1}=\right.$ surface, $A_{2}=$ fond $)$, type de marée $\left(B_{1}=\right.$ morteeau, $B_{2}=$ vive-eau) et date (mois de janvier à décembre), $A B_{i j}$, $A C_{i k}$ et $B C_{j k}$ les interactions deux à deux et $\epsilon_{i j k}$ le résidu.

Le pas de temps des phénomènes biologiques est de l'ordre de la journée. Bien que le temps de réponse des mollusques filtreurs aux fluctuations rapides du milieu n'ait pas été étudié, les données de temps de transit intestinal, entre 2 et $30 \mathrm{~h} \mathrm{chez}$ Mytilus edulis (Bayne et al. 1987) ou chez Crassostrea gigas (Boucaud-Camou et al. 1985; Le Gall, comm. pers.) sont des indicateurs de l'effet intégrateur de l'individu. En fonction de ce pas de temps et de la variabilité du milieu, il est nécessair de reconstituer le cycle des variables hydrologiques, seston e PLG. Un modèle composé d'un facteur autorégressif et d termes non linéaires comprenant les différentes composante des vitesses du vent et du courant selon les orientations est ouest et nord-sud, responsables du transport particulaire et $d$ la remise en suspension, a été utilisé. Le vent marque une ter dance saisonnière, mais son évolution journalière est de tyf stochastique (Bacher 1989). Les mesures de sa direction et $c$ sa vitesse proviennent des enregistrements, toutes les $3 \mathrm{~h}$ et deux stations, fournis par la Météorologie Nationale. Par sou 


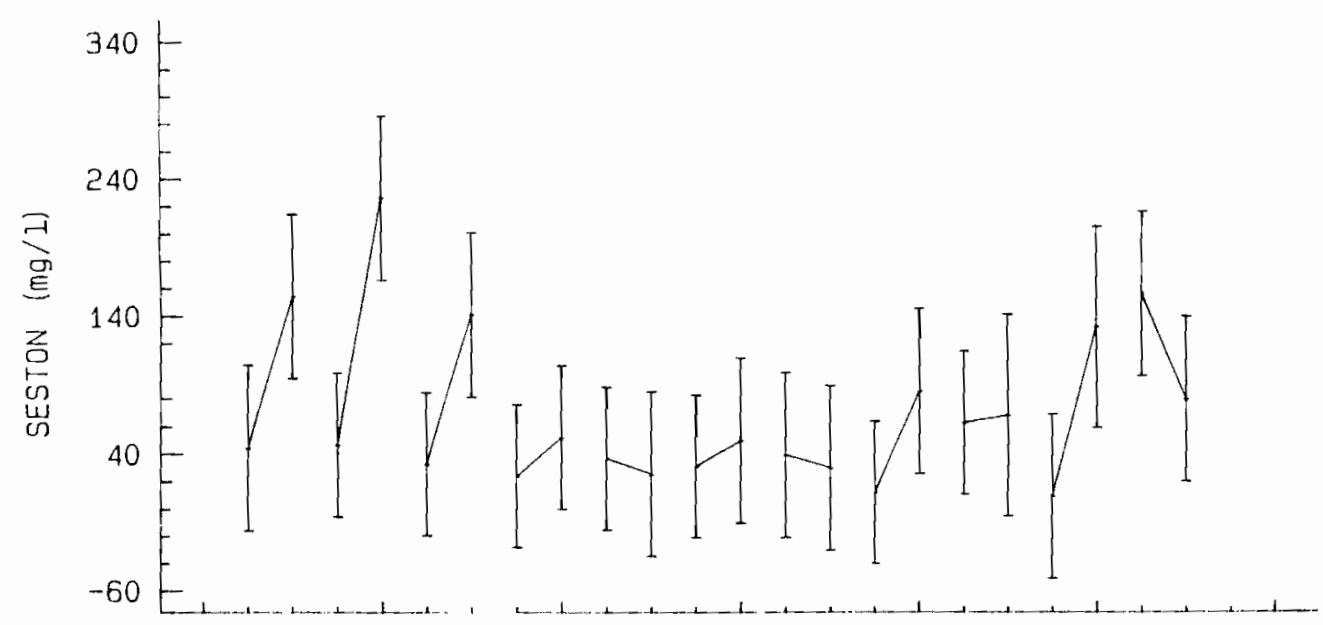

FEV MAR AVR MAI JUI JUI AOU SEP OCT NOV DEC

MOIS/MAREE

d) INIERACTION ENTAE L.FS FACTEURS MOIS ET MAREE FEN 1980

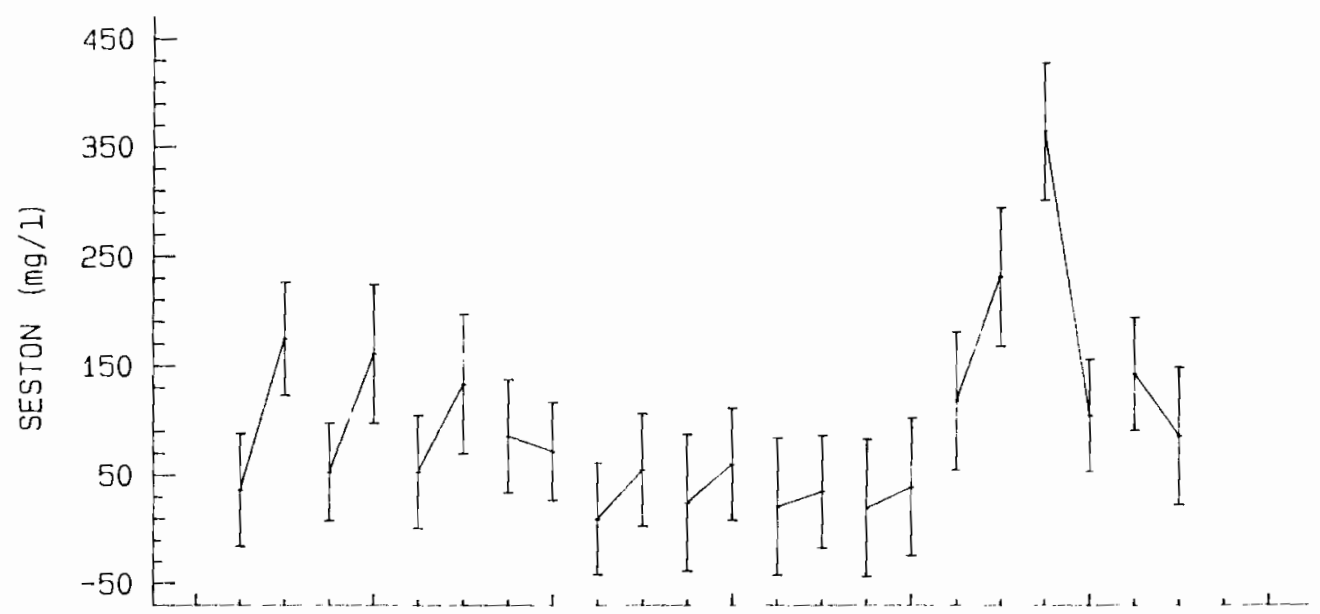

FEV MAR AVR MAI JUI JUI AOU SEP OCT NOV DEC

MOIS/MAREE

FIG. 2. (Fin)

de simplification, ces données ont été moyennées chaque jour. Enfin les courants ont été mesurés aux heures de prélèvement, mais un courant standard (Laboratoire Central Hydraulique de France (LCHF) 1973) a été utilisé dans le modèle, bien qu'il soit sensible au vent et peu variable en fonction du coefficient de marée.

Une fois le modèle calé, une simulation avec un pas de temps de $2 \mathrm{~h}$ a été lancée sur les deux années d'étude. Un programme du Service Hydrographique et Océanographique de la Marine (SHOM), adapté sur compatible IBM/PC, a été utilisé pour prédire la hauteur d'eau toutes les $2 \mathrm{~h}$ en conditions météorologiques normales. Ce même programme a fourni les hauteurs de la colonne d'eau. La moyenne journalière du seston a été déduite des simulations du modèle autorégressif. Le modèle s'écrit

$$
Y_{t}=f\left(Y_{t-1}, Z_{t}, U_{t}, V_{t}, U_{x}, U_{y}, V_{x}, V_{y}, U_{x} \cdot V_{x}, U_{y} \cdot V_{y}, U V\right)
$$

où $f$ est une fonction linéaire de la hauteur $Z$, de la vitesse du vent $U$, de la vitesse du courant mesuré $V$ et de leurs composantes selon les axes ouest-est $(x)$ et sud-nord $(y)$. Les termes non linéaires $U_{x} V_{x}$ et $U_{y} V_{y}$ ont été également introduits.

Modèle de croissance

Le modèle de bilan énergétique s'écrit

(1) $d W / d t=(A-R) \cdot \mathrm{p} \cdot \mathrm{TIM}$

où $W=$ poids sec converti en énergie (Deslous-Paoli 1980), $d W / d t=$ taux de croissance instantanée (joules par heure), $A$ $=$ assimilation et $R=$ respiration, fonctions du seston (variable $S)$, de la somme des équivalents énergétiques des protides, 
Tableau 1. Pourcentages de variation du seston et de PLG expliqués par les facteurs date et coefficient de marée et leur interaction dans les ANOVA par année et hauteur de prélèvement.

\begin{tabular}{lccc}
\hline & & \multicolumn{2}{c}{ Hauteur } \\
\cline { 2 - 3 } Année & Variable & Surface & Fond \\
\hline 1979 & PLG & 74 & 73 \\
& Seston & 61 & 57 \\
1980 & & 83 & 80 \\
& PLG & 82 & 78 \\
\hline
\end{tabular}

lipides et glucides de la colonne d'eau (variable PLG), de la température $T$ et de $W, p=$ proportion d'énergie consacrée à la chair, $1-p=$ proportion consacré à la coquille en fonction de la classe d'âge (Deslous-Paoli 1980) et TIM $=$ fraction de la durée journalière d'immersion. La variable TIM est prédite à l'aide d'une loi empirique calée sur les observations recueillies sur le site et fonction du coefficient de marée.

Peu de travaux sont orientés sur la détermination de la date de ponte et l'intensité de celle-ci. Bayne et al. (1976) et Verhagen (1982) proposent des lois allométriques ou fonction de la production ("scope for growth"). Il est vraisemblable cependant que la température joue un rôle dans le déclenchement de la ponte et dans la gamétogenèse (notion de degrés-hours, Héral et al. 1986b). D'autre part, il apparait que, si la ponte est pratiquement instantanée pour un individu, elle peut s'étaler sur plusieurs jours au niveau de la population. Faute de données, la date de ponte n'est pas prédite mais imposée selon les observations des années 1979-1980. L'intensité de la ponte est représentée comme la perte d'une fraction du soma, fonction du poids sec à la date de ponte, et étalée dans le temps de sorte que l'effort de reproduction total corresponde aux données de la littérature pour les classes d'âge 1 et 2 . La croissance individuelle modélisée représente l'évolution d'un individu moyen sans que soit prise en compte la variabilité de la population. Le terme de respiration est tiré de Boukabous (1983) et ne dépend que du poids sec (fonction allométrique d'exposant $R_{1}$ ) et de la température $T$ (loi exponentielle) :

$$
R=R_{0} \cdot W \cdot \operatorname{EXP}\left(R_{1} \cdot T\right)
$$

Le terme d'assimilation est plus complexe. Le fonctionnement du bivalve filtreur se décompose en trois étapes : filtration, ingestion et assimilation. Pour l'huittre, la filtration est une relation allométrique indépendante des conditions du milieu (Deslous-Paoli et al. 1987). Le taux d'ingestion a été explicité par Bayne et al. (1976) puis Verhagen (1982) pour Mytilus edulis et traduit le fait qu'à charge sestonique croissante, une part croissante de particules consommées est évacuée sous forme de pseudofèces (encombrement des palpes labiaux). Cette action inhibitrice de la charge sestonique $S$ (milligrammes par litre) est formulée ici par une fonction inverse :

$$
t_{i}=\frac{1}{S+S_{0}}
$$

Le taux d'assimilation $t_{a}$ dépend de la densité d'organique ingérée (rapport organique/minéral), de la température (Dame et al. 1980) et de la qualité de la matière organique (Epifanio
TABLEAU 2. Résultats des régressions linéaires pas à pas du seston total et de la variable PLG en fonction des vitesses de vent et de courant, de la hauteur d'eau et d'un terme autorégressif.

Variable $\quad \begin{gathered}\text { Coefficient } \\ \text { de regression }\end{gathered} \quad$ Probabilité

a) Variable $Y=$ seston total; $R$ multiple $=0,774$

$\begin{array}{lrc}Y(t-1) & 0,522 & <10^{-3} \\ \text { Hauteur } & -8,400 & 0,05 \\ \text { Courant } & 114,024 & 0,01 \\ \text { Vent } & 9,852 & <10^{-3}\end{array}$

b) Variable $Y=P L G ; R$ multiple $=0,791$

$Y(t-1)$

0,697

$<10^{-3}$

1979). La relation entre le taux d'assimilation et les variables du milieu est de la forme

$$
t_{a}=\mathrm{PLG} \cdot \frac{T-T_{0}}{T_{1}-T_{0}} \cdot \operatorname{EXP}\left(\frac{T_{1}-T}{T_{1}-T_{0}}\right)
$$

où $T$ désigne la température et PLG la concentration de nourriture convertie en énergie (joules par litre). L'expression de l'influence de la température traduit son effet inhibiteur pour $T>T_{1}$ ou $T<T_{0}$ (Newell 1979). La fonction d'assimilation s'écrit finalement

$$
A=F_{1} \cdot W \cdot t_{i} \cdot t_{a} \cdot
$$

Le temps d'immersion est directement relié au coefficient de marée $C$ à l'aide de la loi empirique, propre au site étudié :

$$
\begin{aligned}
& \text { TIM }=p+r \cdot(C-q)^{2}, \quad \text { si } C>70 \\
& \mathrm{TIM}=24, \quad \text { si } C<70
\end{aligned}
$$

où $p, q$ et $r$ sont estimés à partir des mesures in situ. La température est interpolée linéairement en fonction du temps entre les dates de prélèvement.

Les paramètres $F_{1}, F_{2}, T_{0}, T_{1}$ et $S_{0}$ sont estimés par optimisation. L'exposant de l'allométrie $F_{2}$ est de l'ordre de 0.75 (Fiala-Médioni et Copello 1985). L'algorithme utilisé est le simplex (Nelder et Mead 1965) adapté par Schnute (1982), basé sur la minimisation de la somme des carrés des écarts entre observations et prédictions, grâce à une méthode itérative. A partir du lot de paramètres ainsi trouvé, l'équation 1 permet de simuler la croissance sur 2 ans. La régression entre observations et valeurs simulées, en tenant compte du nombre de degrés de liberté nécessaires à l'estimation des paramètres, mesure l'adéquation du modèle.

\section{Modèle monoboite}

Le modèle précédent sert à illustrer, de façon théorique, la notion de capacité trophique. En supposant les concentrations des divers types particulaires homogènes dans le bassin, et connaissant le temps de résidence moyen des masses d'eau, l'estuaire peut être considéré comme un "raceway" dans lequel la population d'huîtres est soumise aux mêmes conditions de nutrition. Notons $C(t)$ la concentration énergétique de nourriture (joules par litre) dans la boite, $C_{0}(t)$ la concentration en amont (limite) à l'instant $t, V$ son volume (mètres cube) et $F$ son débit (mètres cube par jour) d'entrée (ou de sortie), $N_{i}$ le 


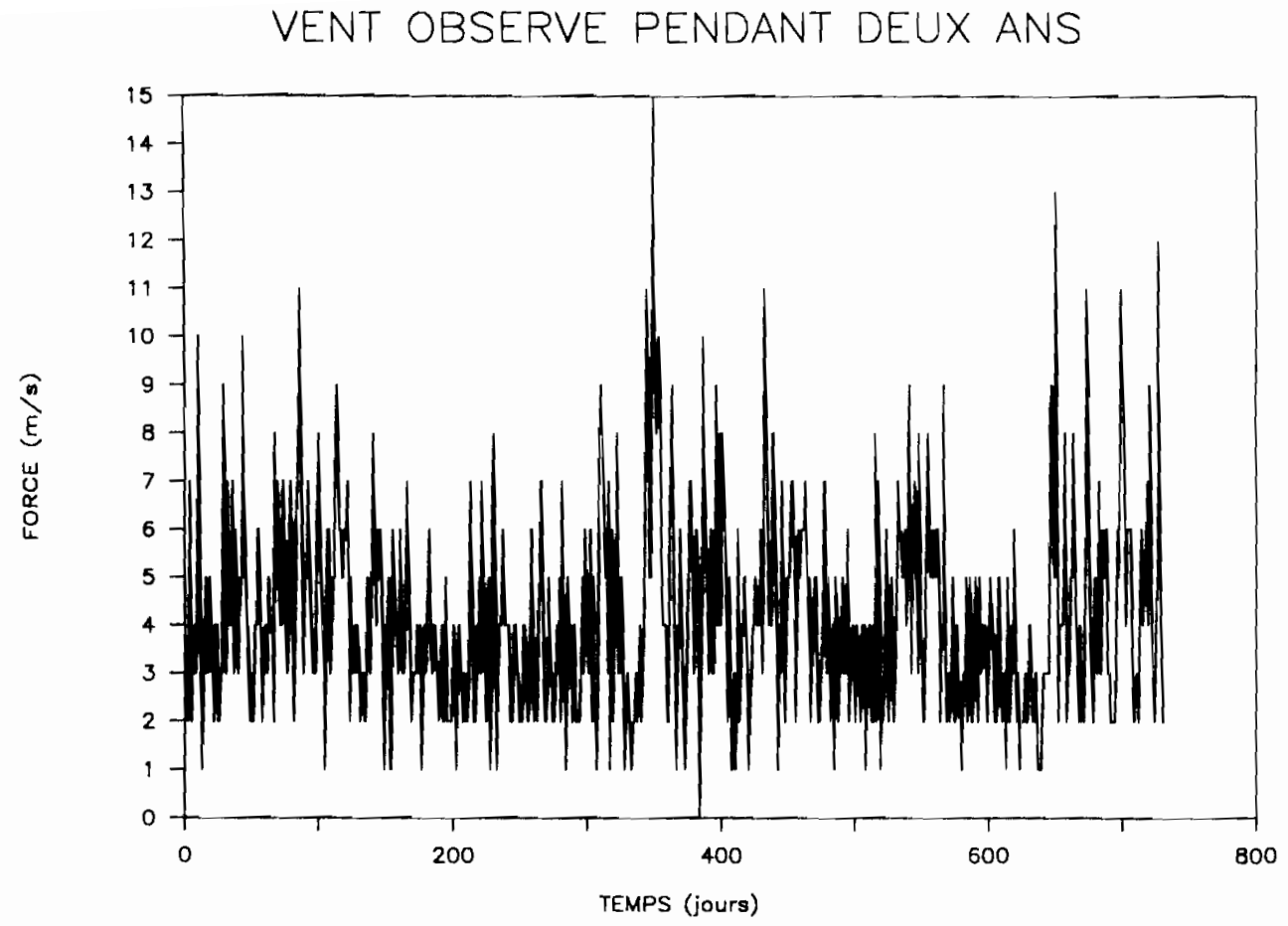

Fig. 3. Série observée de la force du vent en 1979-1980 introduite dans le modèle de reconstitution des séries temporelles des seston et PLG (source Météorologie Nationale). Le vent agit sur la concentration particulaire à la fois en modifiant le transport par les courants et en contribuant à la remise en suspension du fond.

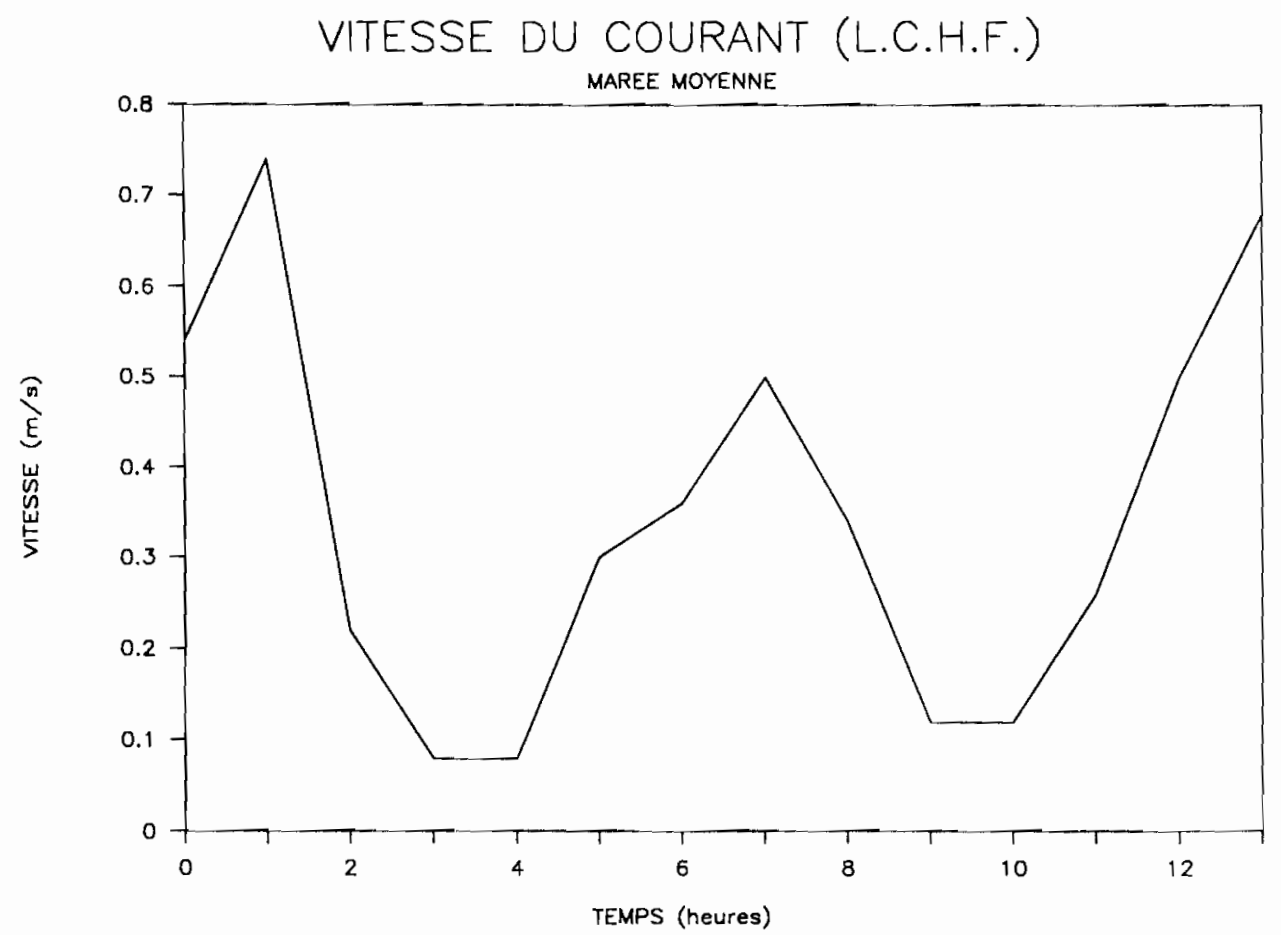

FIG. 4. Force du courant observé pendant un cycle de marée moyenne en partant de l'heure de basse mer (LCHF 1973).

nombre d'individus de la classe d'âge $i(i=1,2)$, le système s'écrit

$V d C / d t=F \cdot\left(C_{0}-C\right)-\sum_{i} A_{i} \cdot N_{i}$, équation de conservation

$d W_{i} / d t=\left(A_{i}-R_{i}\right) \cdot p_{i} \cdot$ TIM, équation de croissance où $A_{i}=$ assimilation (joules par heure), $R_{i}=$ respiration (joules par heure) et $W_{i}=$ poids (joules) individuels pour les classes 1 et 2 (cf. équation 1).

Pour simplifier, la donnée limite $C_{0}(t)$ sera représentée par la série utilisée dans le modèle énergétique. La variable $S(t)$ est 
a) SESTON EN 1979

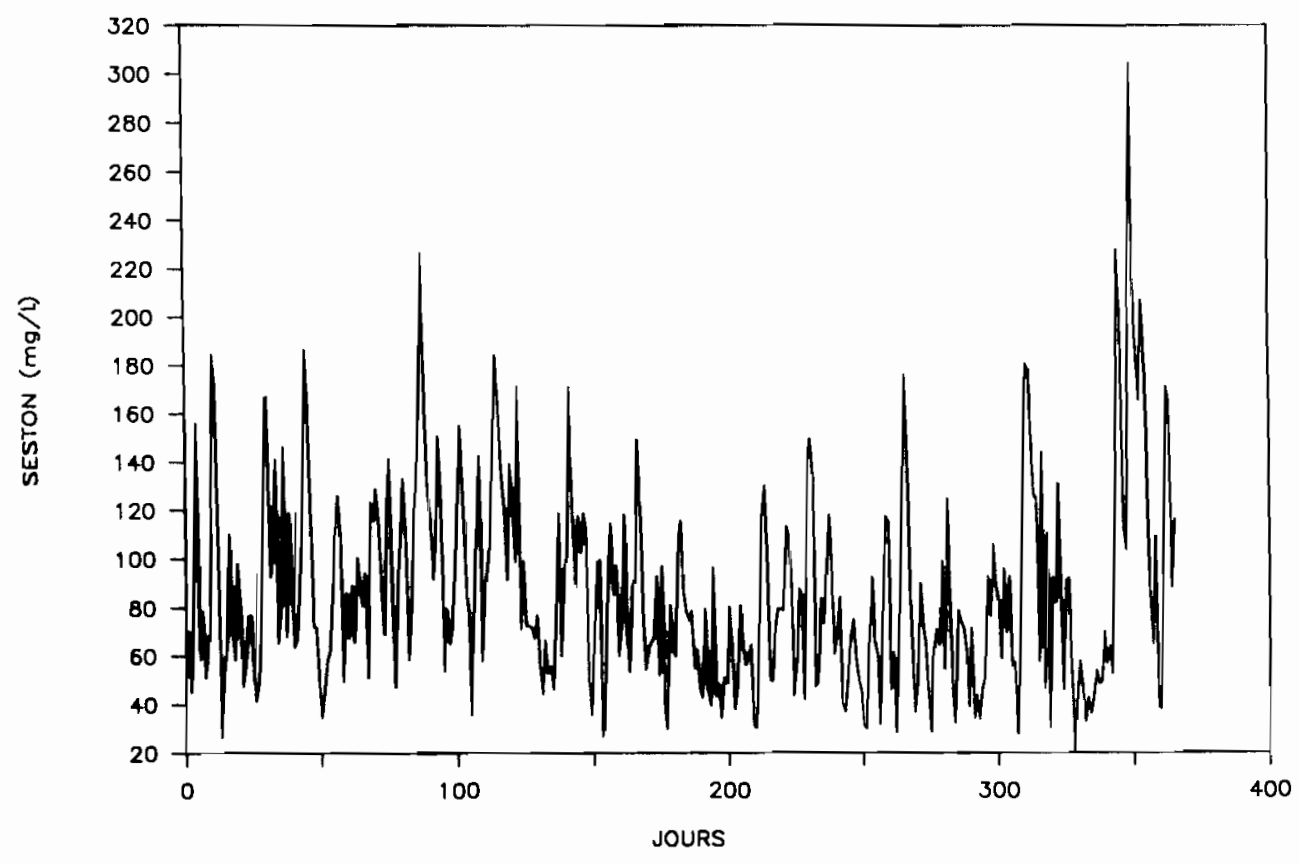

b) SESTON EN 1980

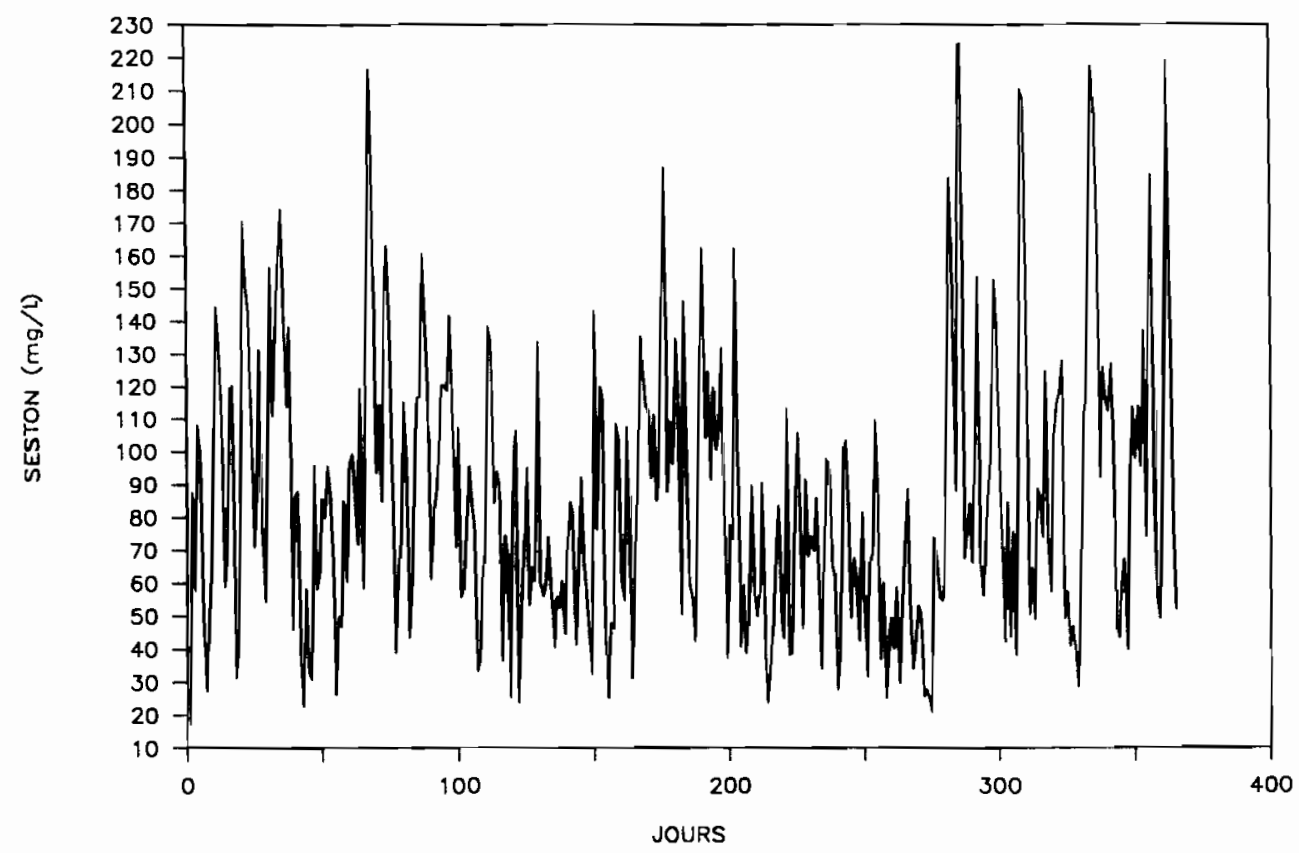

FiG. 5. Séries journalières simulées du seston et de la variable PLG (somme des équivalents énergétiques des concentrations en protides, glucides et lipides particulaires de la colonne d'eau) en 1979 et 1980 en prenant en compte la force du vent, la vitesse du courant et la hauteur d'eau. Ces simulations font ressortir l'évolution annuelle liée à la saisonnalité et la forte variabilité journalière due au vent. a) Seston en 1979; b) seston en 1980; c) PLG en 1979; d) PLG en 1980.

représentée par la série vue plus haut en supposant que les pseudofèces et fèces sont intégralement remis en suspension.

Le volume moyen du Bassin a été calculé d'après les sorties en hauteur d'eau d'un modèle courantologique réalisé par le LCHF (1979), seion un maillage rectangulaire pour deux coefficients de marée types. Le débit est le débit résiduel moyen estimé d'après les mesures et calculs effectués en morte-eau et vive-eau par le LCHF (1973). La croissance finale et le maximum de croissance (précédant la ponte) sont fonctions des effectifs $N_{i}(i=1,2)$. Le stock d'huîtres, estimé par échantillonnage annuel depuis 1984 , a été converti en effectifs pour les classes d'âge 1 et 2 ans (Bodoy et al. 1987). Des simulations sont effectuées avec différents niveaux d'effectifs de chaque classe d'âge. Trois types de sorties sont représentés graphique- 
c) PLG EN 1979
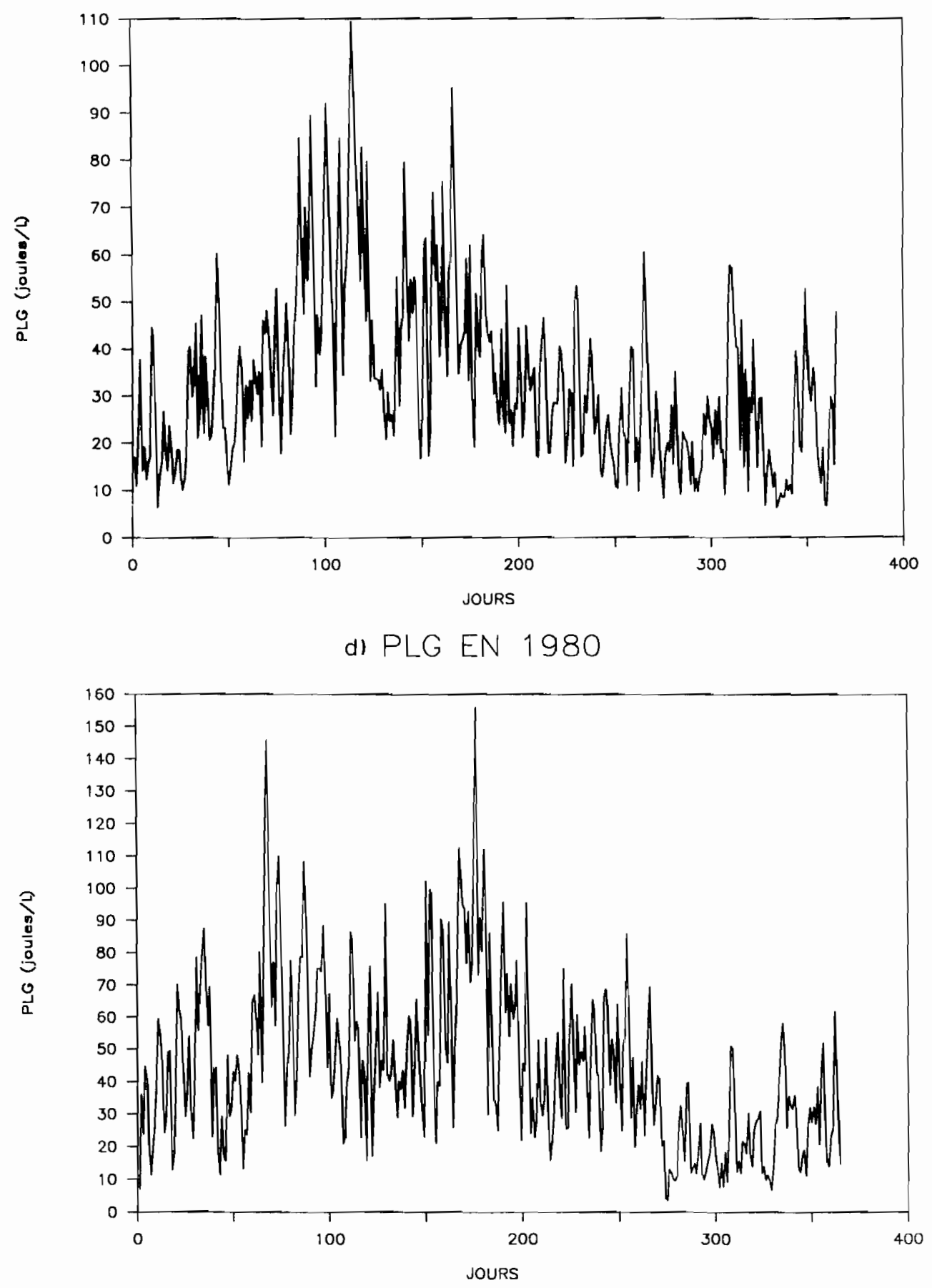

FIG. 5. (Fin)

ment afin d'illustrer la relation effectif/performance de croissance :

a) poids individuel moyen en fin de croissance.

b) production totale annuelle de la classe d'âge $1\left(\mathrm{PT}_{1}\right)$ et production totale des classes 1 et $2\left(\mathrm{PT}_{2}\right)$.

$$
\begin{aligned}
& \mathrm{PT}_{1}=N_{1} \cdot W_{1} \\
& \mathrm{PT}_{2}=N_{1} \cdot W_{1}+N_{2} \cdot\left(W_{2}-W_{1}\right)
\end{aligned}
$$

avec $N_{1}$ (resp. $N_{2}$ ) = effectif de la classe d'âge 1 (resp. 2) et $W_{1}$ (resp. 2$)=$ poids final de la classe 1 (resp. 2 ). c) proportion de nourriture potentielle assimilée en un an par la population.

$$
\mathrm{PA}=\frac{\sum_{i} \int_{t} N_{i} \cdot A_{i}(t) d t}{F \cdot \int_{t} C_{0}(t) d t}, \quad i=\text { classe d'âge. }
$$

\section{Résultats}

Variabilité du milieu

L'ANOVA sur les différents facteurs de variabilité naturelle 


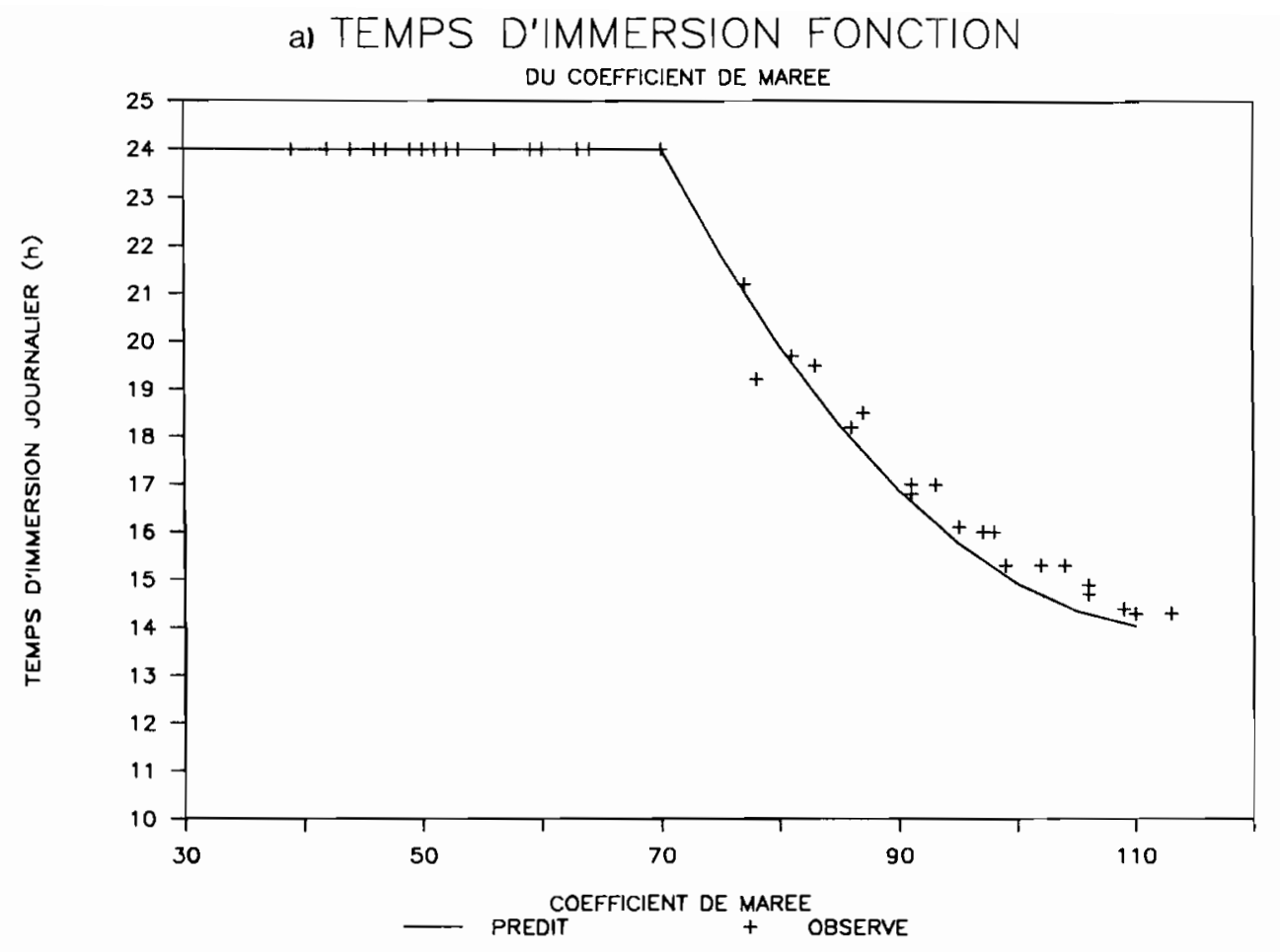

b) TEMPS D'IMMERSION 1979-1980

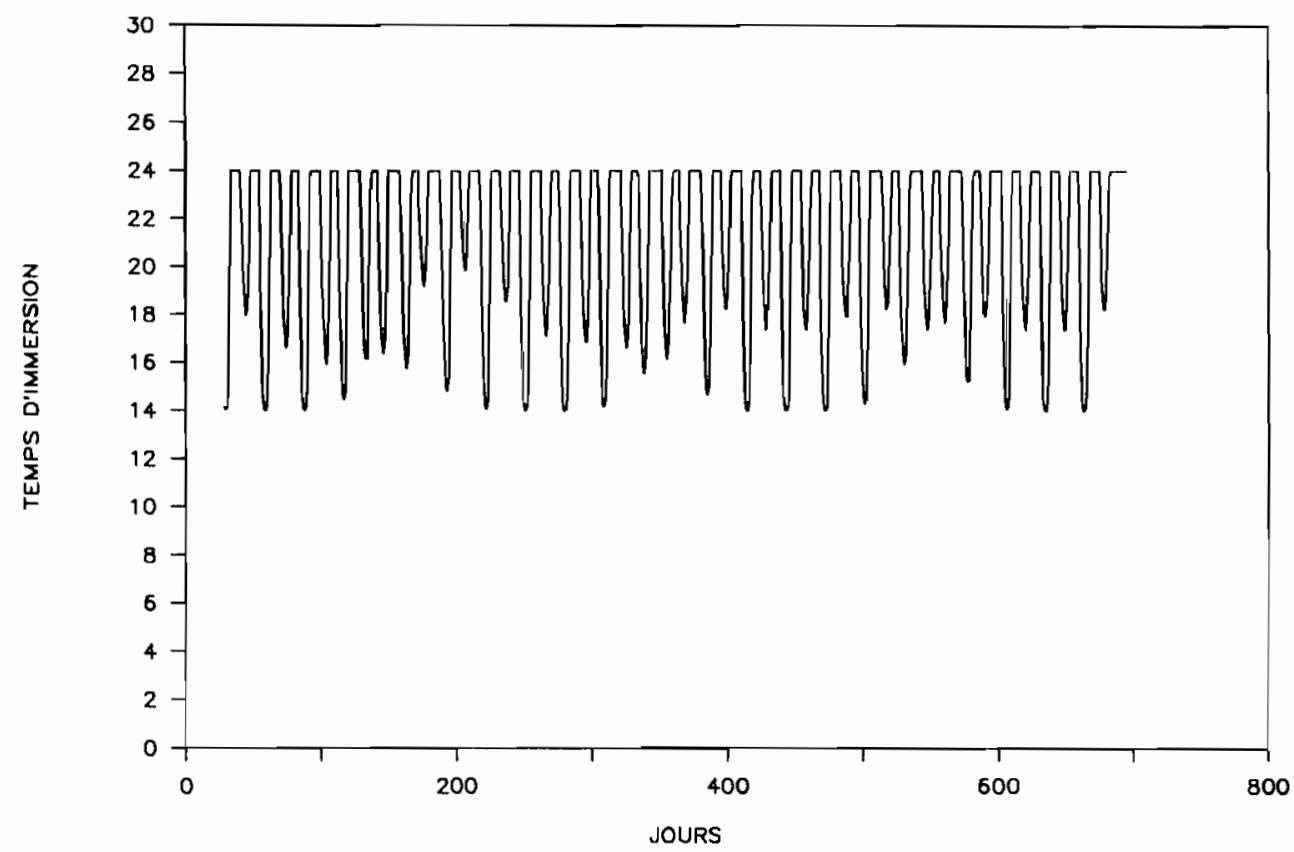

FIG. 6. Variables forçantes utilisées dans le modèle de bilan énergétique (années 1979 et 1980). a) Loi empirique du temps d'immersion en fonction du coefficient de marée ( + , observations: - prédictions); b) Temps d'immersion sur 2 ans; c) température mesurée pendant 2 ans.

du seston (variable $S$ ) et de la variable PLG permet de hiérarchiser les contributions de chaque facteur à la variabilité observêe. Quelles que soient l'année et la variable étudiée, il y a interaction entre la date et le coefficient de marée (probabilité $<10^{-4}$ ). Afin d'étudier cette interaction, des ANOVA ont été réalisées sur les facteurs date et type de marée pour chaque modalité de hauteur de prélèvement. La représentation graphique des moyennes et intervalles de confiance montre une différence entre les types de marée pour les mois de février, mars et avril pour les deux variables étudiées en 1979 et 1980 (fig. 2). En général, cette différence est moins nette le reste de l'année. Il peut arriver qu'elle s'inverse lors de conditions météorologiques particulières (tempête en novembre 1980). On peut donc affirmer que le cycle morte eauvive eau explique une part, fonction du temps mais importante, de la variabilité observée. Le bloom phytoplanctonique explique la tendance saisonnière. La combinaison des effets saisonnier et tidal et de leur interaction explique environ $80 \%$ de la 
c) TEMPERATURE $1979-1980$

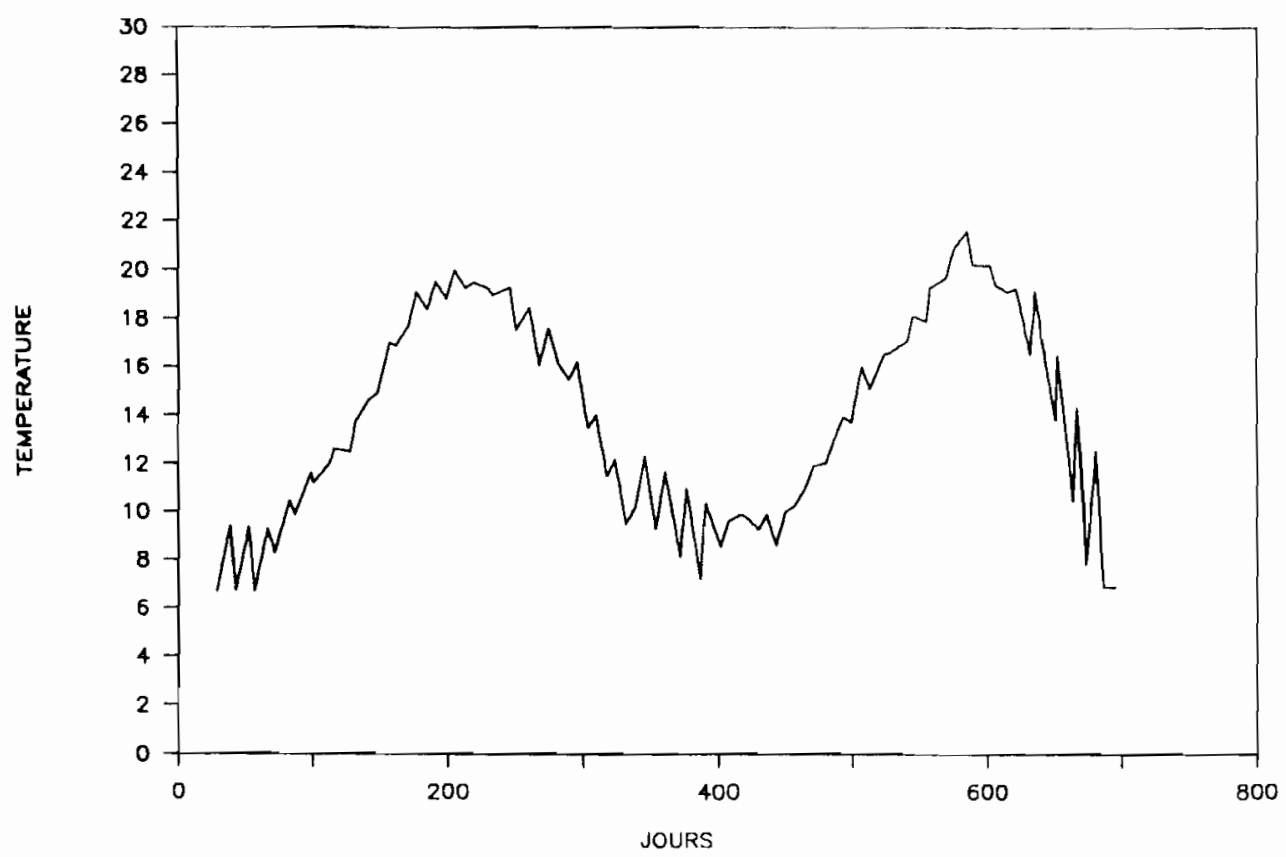

FIG. 6. (Fin)

variabilité totale (tableau 1). La variabilité résiduelle intègre l'erreur du modèle, la variabilité horaire.

Ces résultats justifient l'utilisation d'un modèle prédictif incluant principalement des fonctions périodiques correspondant aux sources majeures de fluctuations et un terme susceptible d'expliquer une partie de la variance résiduelle sous forme d'un processus stochastique. Courants, hauteurs d'eau et vent sont donc introduits dans un modèle mixte comprenant des termes linéaires et des effets multiplicatifs.

La sélection des variables les plus significatives dans l'explication des variables $S$ (seston total) et PLG (somme des concentrations de protides, lipides et glucides converties en énergie) montre que les termes non linéaires jouent un rôle toujours négligeable (tableau 2). Par contre, un gain notable de signification est obtenu grâce au terme autorégressif. Le coefficient de détermination, qui mesure le pourcentage de variance expliquée, atteint ainsi $60 \%$ pour le seston total. Pour la variable PLG, aucun facteur physique n'est retenu et le terme autorégressif explique à lui seul $62 \%$ de la variance. Dans l'optique prédictive adoptée jusqu'à présent, il a semblé judicieux, bien que moins rigoureux, de calculer le cycle de la variable PLG à partir du cycle de la variable $S$, corrigé d'un facteur estimé mensuellement par la moyenne des rapports observés PLG/S.

La fonction de régression obtenue pour le seston total a été utilisée pour reconstituer le cycle annuel avec un pas de temps journalier en fonction du vent (fig. 3) et du courant (fig. 4). Initialisée par une valeur de seston total arbitraire, une série a été simulée sur les deux années 1979-1980. Les séries des moyennes journalières ont été ensuite calculées pour le seston total et la variable PLG (fig. 5). Les valeurs extrêmes de seston total (supérieures à $100 \mathrm{mg} / \mathrm{L}$ ) sont mal reproduites. Elles correspondent à des phénomènes accidentels (tempêtes) à l'origine de remises en suspension massives et de courte durée. Leur probabilité d'occurrence est faible et peut être considérée comme dépendant de la saison. Elle est ainsi plus forte en hiver et à la fin de l'automne, périodes correspondant à un amaigrissement relativement faible des huîtres. Ces accidents météorologiques apparaissent parfois en période de croissance mais leur courte durée, liée à une resédimentation rapide, permet de tempérer leur influence sur la croissance des huîtres.

\section{Modèle énergétique}

La fig. 6 montre la loi du temps d'immersion en fonction du coefficient de marée et l'évolution du temps d'immersion qui en a été déduite au cours du temps. La variation journalière de la température sur 2 ans est représentée sur la fig. 6c. L'optimisation par la méthode du simplex permet d'estimer les paramètres inconnus du modèle de croissance. La comparaison des poids secs simulés et observés (fig. 7) après calage sert à valider le modèle. La pente de la régression est voisine de 1 (à $5 \%$ ) et l'ordonnée à l'origine de 0 (à $5 \%$ ). Le nombre de degrés de liberté est égal à $N-p-1=20(N=$ nombre d'observations $=26, p=$ nombre de paramètres estimés grâce aux mêmes observations $=5$ ).

Le modèle monoboite théorique illustre la relation effectifperformance de croissance. Le temps de résidence est égal au rapport $V / F$. Le volume moyen $V$ prend la valeur $675 \times 10^{6} \mathrm{~m}^{3}$. Le débit $F$ est de l'ordre de $200 \times 10^{6} \mathrm{~m}^{3} /$ jour en coefficient de vive-eau et $100 \times 10^{6} \mathrm{~m}^{3} /$ jour en morte-eau. Le temps de résidence moyen adopté est donc de 4,5 jours. Vingt simulations ont été réalisées pour des effectifs variables en prenant comme hypothèse que la quantité de nourriture disponible $C_{0}(t)$ à la limite du modèle est indépendante de l'état du stock. Les productions, les poids en fin de croissance et les proportions de nourriture assimilée sont reportés en fonction de l'effectif (fig. 8). La pente $d Y / d X$ de chaque courbe permet de calculer la sensibilité $s$ de la variable expliquée à une variation de l'effectif au voisinage de l'effectif actuel $X_{0}$ :

$$
s=\frac{X_{0}}{Y_{0}} \cdot \frac{d Y}{d X}
$$


a) SIMULATION DU POIDS SEC INDIVIDUEL

EN $1979-1980$

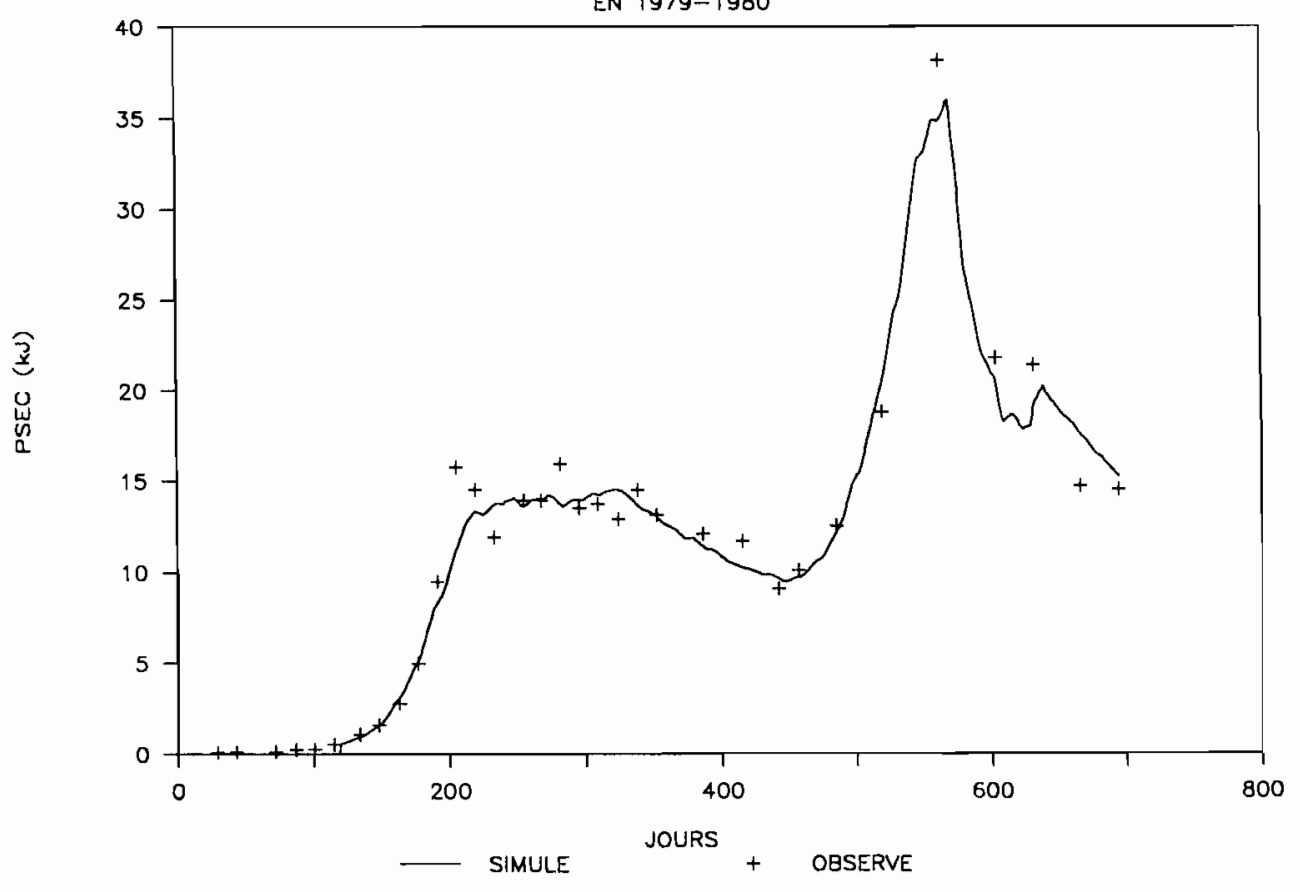

b) REGRESSION POIDS SECS SIMULE / OBSERVE

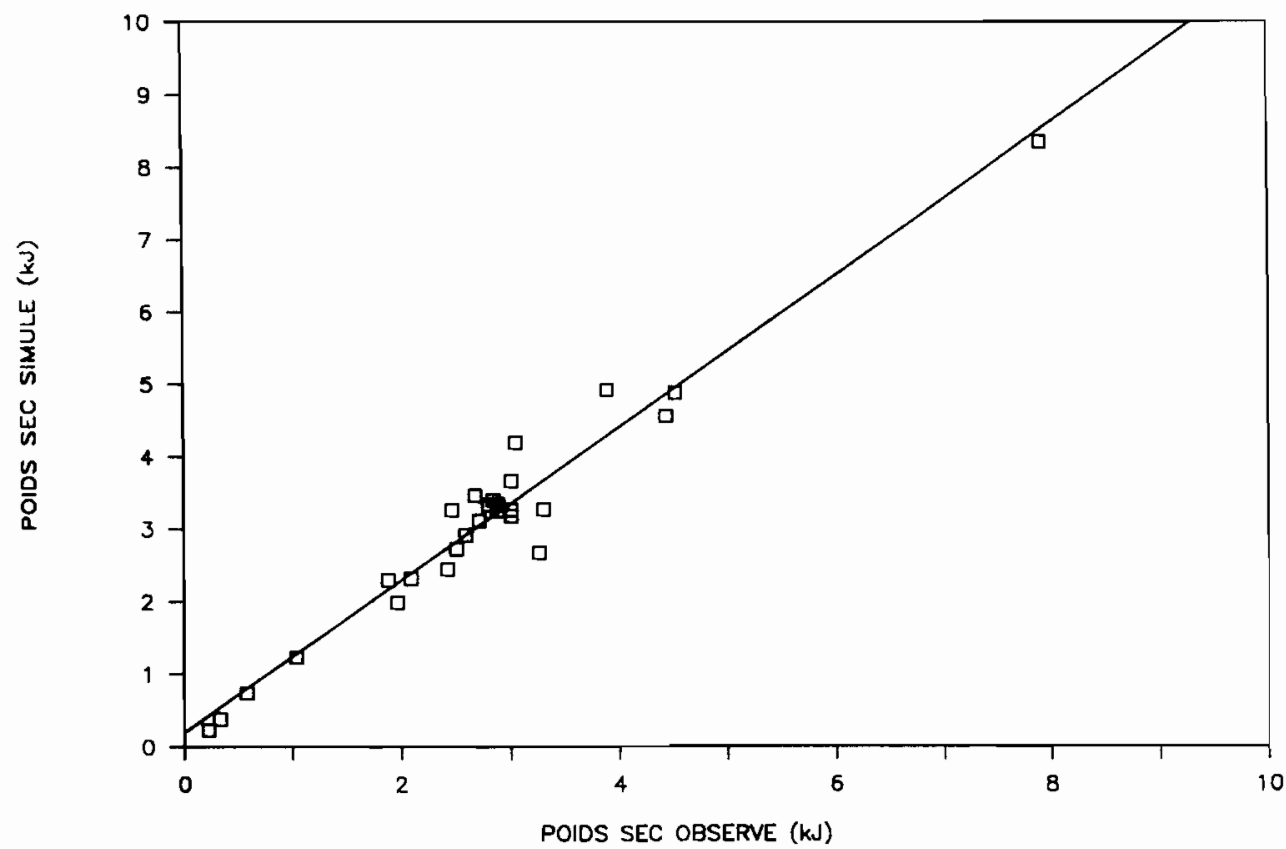

FIG. 7. Résultats de l'estimation des paramètres du modèle de bilan énergétique de Crassostrea gigas. a) Simulation pendant 2 ans du poids sec individuel moyen converti en energie $(-)$ et comparaison avec les valeurs observées $(+)$. La croissance au début de la deuxième année est compensée par une ponte estivale très importante, suivi d'un amaigrissement automnal et hivernal : $F_{1}=24,32, F_{2}=$ $0,602, S_{0}=12,22, R_{0}=1,746, R_{1}=1,3, R_{2}=0,1, T_{0}=9,14, T_{1}=23,80$. b) Régression entre les valeurs simulées et observées du poids sec individuel moyen : $Y=a X+b, N=26, p=5$; $a=$ $1,055, \sigma(a)=0,047 ; b=0,19, \sigma(b)=0,15$.

où $Y_{0}$ est la valeur de la variable expliquée pour l'effectif $X_{0}$. La sensibilité de la proportion assimilée est égale à 0,78 , très proche des sensibilités des productions $\mathrm{PT}_{1}$ et $\mathrm{PT}_{2}(0,83$ et 0,84 respectivement). Pour les poids en fin de croissance, les sensibilités valent $-0,16$ pour les deux classes d'âge. Une aug- mentation du stock se traduit donc par une diminution sensible des performances de croissance.

\section{Discussion}

Le présent modèle permet de présenter le problème de capacité trophique d'un site côtier consacré à la conchyliculture. Par 


\section{a) RELATION EFFECTIF-POIDS FINAL}
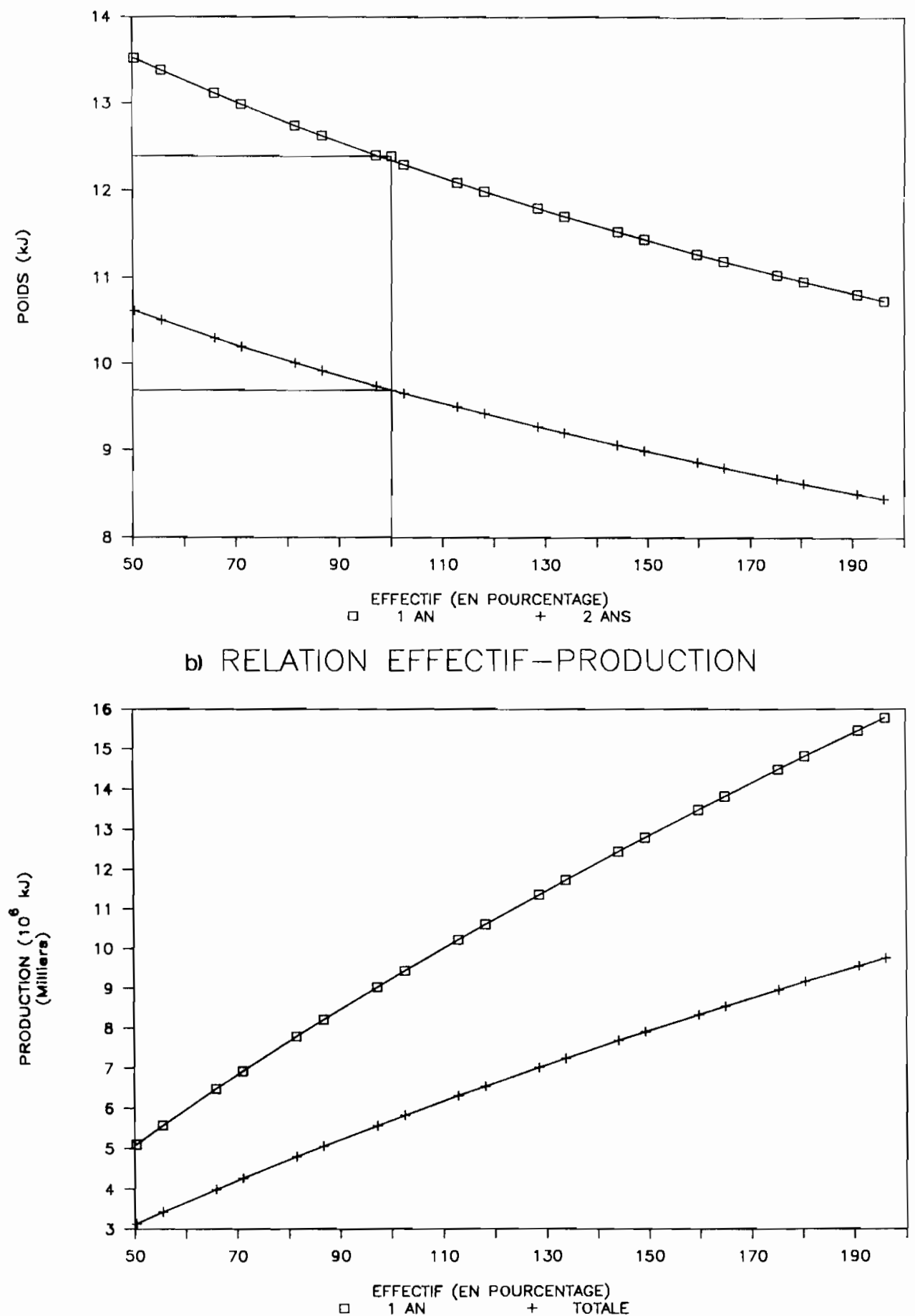

Fig. 8. Influence de l'effectif de la population de Crassostrea gigas sur les résultats de croissance en utilisant un modèle monoboite. La variation de l'effectif est exprimée en pourcentage de l'effectif observé en 1986. La compétition pour la nourriture aboutit à une diminution des performances individuelles de croissance lorsque la quantité d'huîtres augmente (a). Si le nombre d'huîtres est multiplié par 4, la production totale (b) et le pourcentage de nourriture utilisée pour la croissance (c) sont ainsi multipliés par un facteur 3. a) Poids sec individuel converti en énergie du bout de 1 an ( $\square$ ) et 2 ans de croissance $(+)$; b) production pour la classe d'âge $1(\square)$ et la population totale $(+)$; c) pourcentage de nourriture potentielle asimilée par la population par rapport aux flux entrant dans le bassin.

rapport aux études existantes (Incze et al. 1981; Lutz 1980; Verhagen 1982), il donne une idée de la compétition intraspécifique au niveau macroscopique, bien que certains auteurs (Peterson et Black 1987) insistent sur l'existence d'une compé- tition au niveau local. L'écriture d'une équation de bilan énergétique permet d'aller plus loin que les études plus classiques des relations trophiques dont le but est d'étudier les performances de croissance en fonction des paramètres du milieu 


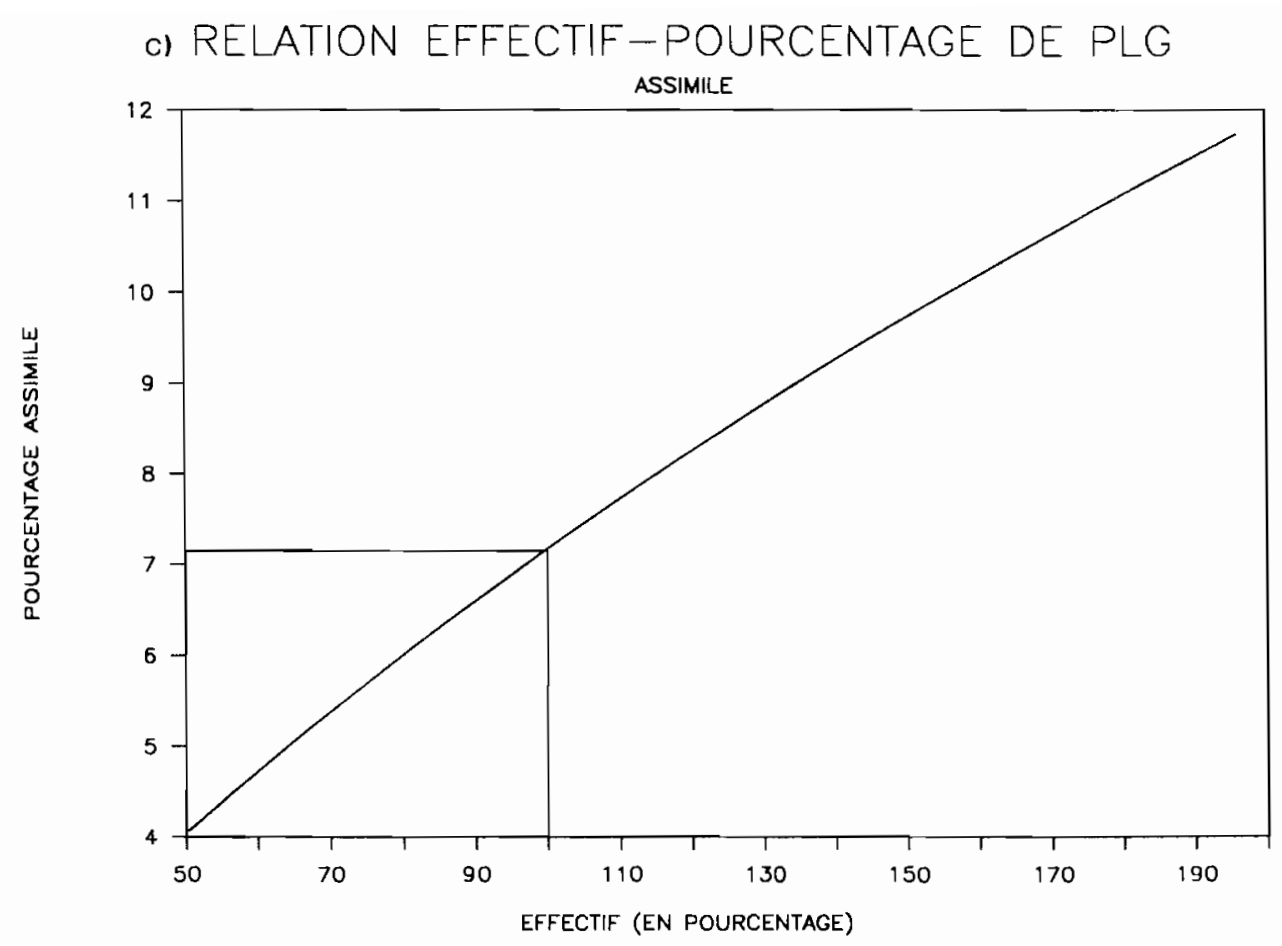

FIG. 8. (Fin)

(Goulletquer et Bacher 1989). Ce modèle indique ainsi que, même si le pourcentage d'énergie assimilée paraît faible (environ $7 \%$ ), la déplétion de nourriture est déjà suffisante pour qu'une augmentation des effectifs se traduise par une perte de croissance équivalente. La sensibilité de la production, inférieure à 1 , montre le ralentissement de l'augmentation de production résultant de l'accroissement du stock. La concavité de la courbe de production illustre la non-linéarité de la réponse de la population à une variation des effectifs. C'est la définition même d'un milieu dont la nourriture est limitante. Cette conclusion remet ainsi en cause les calculs de bilan et de pourcentage d'énergie utilisée au niveau local sans tenir compte du temps de résidence des masses d'eau (Héral et al. 1983a). C'est donc bien le phénomène de transport particulaire qui détermine la capacité trophique du Bassin. Il parait ainsi probable que des différences spatiales de croissance peuvent être également expliquées par l'appauvrissement des masses d'eau transitant dans le bassin, au fur et à mesure de leur utilisation par les filtreurs. L'homogénéisation des masses d'eau dans les estuaires macrotidaux montre cependant qu'il n'y a pas déplétion de nourriture à une échelle spatiale inférieure au kilomètre. L'échelle spatiale compatible avec la perte de nourriture due à la consommation dépend finalement de l'amplitude de l'excursion de marée, largement responsable du confinement des masses d'eau. Le couplage d'un modèle physique de transport particulaire et du modèle de croissance doit permettre d'approfondir l'étude du lien stock-performance de croissance. Il est déjà clair qu'il existe un gradient de croissance nord-sud (A. Bodoy, comm. pers.) et que ce gradient est corrélé à l'écoulement résiduel des masses d'eau transitant dans le bassin (Bacher 1989). La variabilité spatiale des performances de croissance ne peut être approchée que par un modèle associant croissance et nourriture disponible selon un découpage spatial du Bassin. De cette étape résulte la possibilité d'une gestion des stocks d'huîtres et de leur répartition en fonction d'un objectif de croissance à déterminer.
Plusieurs types de problèmes ont été rencontrés au niveau de la modélisation. La difficulté à trouver des données bibliographiques quantifiant les réponses des différentes fonctions d'ingestion et d'assimilation constitue un obstacle certain à la robustesse du modèle. Il est clairement démontré, par des suivis in situ et des méthodes de régression (Brown 1988), que température et nourriture contrôlent le bilan de croissance. Cependant, la formulation de la réponse des mécanismes de filtration, ingestion, assimilation et respiration en fonction de ces variables ne fait pas l'unanimité. Rodhouse et O'Kelly (1981) observent ainsi une variation linéaire du taux de filtration de Crassostrea gigas en fonction de la température sans influence de la concentration particulaire. Gerdès (1983) au contraire constate une régulation de la filtration en fonction de la concentration algale. Une autre difficulté provient de ce que les expériences de nutrition (Epifanio 1979; Urban et al. 1983) ne visent pas à reproduire les conditions naturelles caractérisées par une composition et une proportion variable de matière organique particulaire et une turbidité relativement élevée. Aussi, la calibration réalisée sur les données environnementales ne permet pas de généraliser le modèle à un site trop différent de celui étudié. Le domaine de validité du modèle ne s'étend ainsi qu'aux sites de caractéristiques hydrobiologiques comparables. Cependant, le bassin de Marennes-Oléron est un système macrotidal marqué par des cycles de marée très accentués. Malgré un temps de résidence moyen de 5 jours, l'amplitude de l'excursion de marée et l'existence d'estrans de surface importante justifient l'idée de masses d'eau homogènes à l'échelle macroscopique (quelques kilomètres). La situation centrale du point d'étude choisi garantit sa représentativité de l'ensemble du Bassin (Héral et al. 1983b). Il reste néanmoins souhaitable d'intégrer dans cette démarche les résultats de plans d'expérience dans lesquels les paramètres sont contrôlés (Raillard, en prép.).

La sensibilité des séries temporelles de seston et de PLG aux accidents météorologiques, à l'échelle de temps des méca- 
nismes biologiques, peut être expliquée par l'influence du vent sur le transport particulaire et la remise en suspension du sédiment. L'importance de ces phénomènes et la difficulté de reconstituer la série de la nourriture potentielle mesurée par la somme des variables protides, lipides et glucides montrent l'effort à fournir en matière d'échantillonnage en milieu estuarien. La différence entre les deux variables expliquées est à rechercher vraisemblablement dans l'existence de cycles organiques saisonniers, identifiés par Héral et al. (1983b) sous la forme d'apports phytoplanctoniques ou détritiques d'origine océanique (été) ou continentale (hiver). Dans la perspective du couplage d'un modèle de transport et du modèle de bilan énergétique, le besoin de séries aux limites du modèle spatial renforce l'idée d'un accroissement de l'effort d'échantillonnage. La période hivernale expliquant peu les variations de croissance, l'effort en question est à moduler selon les saisons.

\section{Références}

Bacher, C. 1989. Étude de la capacité trophique du bassin de Marennes Oléron : utilisation d'un modèle couplé de transport particulaire et de crois sance de l'huître Crassostrea gigas. Thèse dr., Université de Brest, France. $149 \mathrm{p}$.

Bayne, B. L., A. J. S. Hawkins et E. NavarRo. 1987. Feeding and digestion by the mussel Mytilus edulis L. (Bivalvia: Mollusca) in mixtures of sil and algal cells at low concentrations. J. Exp. Mar. Biol. Ecol, 111: 122.

Bayne, B. L., J. Widdows et R. J. Thompson. 1976. Physiological integrations. Dans B. L. Bayne [éd.] Marine mussels : their ecology and physiology. IBP $\mathrm{n}^{0} 10$. Cambridge University Press, Cambridge. $506 \mathrm{p}$.

Bodoy, A., C. BACHER ET P. GEAiron. 1987. Estimation des stocks d'huîtres cultivées dans le bassin de Marennes-Oléron en 1986. Rapp. IFREMER, DRV 87-005 RA/TREM : 71 p.

Bodoy, A. ET M. R. Plante-CunY. I984. Relations entre l'évolution saisonnière des populations de palourdes (Ruditapes decussatus) et celles des microphytes benthiques et phytoplanctoniques (golfe de Fos, France) Haliotis $14: 71-78$

Boucaud-Camou, E., C. Lebesnerais, P. Lubet et I. Lihrmann. 1985. Dynamique et enzymologie de la digestion chez l'huitre Crassostrea gigas (Thunberg). Bases biologiques de l'aquaculture, Montpellier, 1983. IFREMER, Actes de colloques $n^{\circ} 1$ : 75-96.

BouxABous, R. 1983. Étude préliminaire des adaptations écophysiologiques de l'huître Crassostres gigas (Thunberg) dans la lagune de Oualidia. Mémoire de 3ème cycle agronomie, option : halieutique. Institut Agronomique et Vétérinaire Hassan II, Maroc.

BRown, J. R. 1988. Multivariate analyses of the role of the environmental factors in seasonal and site-related growth variation in the Pacific oyster Crassostrea gigas. Mar. Ecol. Prog. Ser. 45 : 225-236.

DAME, R., R. ZINGMaRK, H. Stevenson ET O. Nelson. 1980. Filter feeding coupling between the estuarine water column and benthic subsystems, p. 521-526. Dans V. A. Kennedy [éd.] Proceedings of the Fifth International Estuarine Research Conference.

Deslous-PaOli, J. M. 1980. Contribution à l'étude de la biologie de l'huître Crassostrea gigas Thunberg dans le bassin et les claires de MarennesOléron. Thèse 3ème cycle, Université d'Aix-Marseille II. $121 \mathrm{p}$.

Deslous-Paoli, J. M. et M. Héral 1984. Transferts énergétiques entre la nourriture potentielle disponible dans l'eau d'un bassin ostréicole et l'huître Crassostrea gigas âgée de 1 an. Haliotis $14: 79-90$.

Deslous-Paoli, J. M., M. Héral, P. Goulletquer, W. Boromthanarat, D. Razet, J. Garnier, J. Prou et L. Barlllet. 1987. Evolution saisonnière de la filtration de bivalves intertidaux dans des conditions naturelles. Oceanis $13: 575-579$.

EPIFANIO, C. E. 1979. Growth in bivalve molluscs : nutritional effects of two or more species of algae in diets fed to the American oyster Crassostrea virginica (Gmelin) and the hard clam Mercenaria mercenaria (L.). Aquaculture, $18: 1-12$.

Fiala-MÉDIONi, A. ET M. Copello. 1985. Relations trophiques entre l'huître et le milieu : influence de la concentration et de la taille des particules. Bases Biologiques de l'Aquaculture, Montpellier 1983, IFREMER Actes de Colloques $\mathrm{N}^{\circ} 1: 63-74$

Gerdès, D. 1983. The Pacific oyster Crassostrea gigas. Part I. Feeding behaviour of larvae and adults. Aquaculture $31: 195-219$
Goulleteuer, P. ET C. Bacher. 1989. Empirical modelling of the growth of $R$. philippinarum by means of non-linear regression on factorial coordinates. Aquat. Living Resour. 1 : 141-154.

Harris, J. R. W., A. J. Bale, B. J. Bayne, R. F. C. Mantoura, A. W Morris, L. A. Nelson, P. J. RAdFord, R. J. UnCles, S. A. Weston ET J. WidDows. 1984. A preliminary model of the dispersal and biological effect of toxine in the Tamar estuary, England. Ecol. Model. $22: 253$ 284.

HÉRAL, M. 1985. Evaluation of the carrying capacity of the molluscan shellfish ecosystems, p. 297-318. Dans Shellfish culture Development and Management, Aquaculture. Inemational Seminar in La Rochelle, 4-9 mars 1985, IFREMER edit. Brest.

Héral, M., J. M. Deslous-Paoli et J. Prou. 1986a. Dynamique des productions et des biomasses des huîtres creuses cultivées (Crassostrea angulata et Crassostrea gigas) dans le bassin de Marennes-Oléron depuis un siècle. Note au CIEM, CM 1986/F : 41 .

1988. Approche de la capacité trophique d'un écosystème conchy licole. Note au CIEM, CM 1988/K : 22.

Héral, M., J. M. Deslous-Paolt, J. Prou et D. Razet. 1987. Relation entre la nourriture disponible et la production de mollusque en milieu estuarien variabilité temporelle de la colonne d'eau. Haliotis $16: 149-198$.

héral, M., J. M. Deslous-Paoli, D. Razet et J. Prou. 1984. Essai de mise en évidence in situ de paramètres biotiques et abiotiques de l'eau et de l'interface eau-sédiment intervenant dans la production de l'huître Crassostrea gigas. Oceanis $10: 465-475$.

Héral, M., J. M. Deslous-Paoli et J. M. Sornin. 1983a. Transferts énergétiques entre l'huître Crassostrea gigas et la nourriture potentielle disponible dans un bassin ostréicole : premières approches. Océanis $9: 169-$ 194.

Héral, M., J. Prou et J. M. Deslous-Paoli. 1986b. Influence des facteurs climatiques sur la production conchylicole du bassin de Marennes-Oléron. Haliotis 15 : 193-207.

héral, M. D. Razet, J. M. Deslous-Paoli, J. P. Berthomé et J. Garnier. 1983b. Caractéristiques saisonnières de l'hydrodynamique du complexe estuarien de Marennes-Oléron (France). Rev. Trav. Inst. Pêches marit. $46(2)$ : $97-119,1982$ (1983).

Incze, L. S., R. A. Lutz ET E. True. 1981. Modeling carrying capacities for bivalve molluscs in open, suspended-culture systems. J. World Maricult. Soc. $12(1)$ : 143-155.

KITCHELL, J. F. ET J. E. BRECK. 1980. Bioenergetics model and foraging hypothesis for sea lamprey (Petromyzon marinus). Can. J. Fish. Aquat. Sci. $37: 2159-2168$.

Laboratoire Central d'Hydraulique de France. 1973. Étude des phénomènes régissant le bassin ostréicole de Marennes-Oléron. Rapport de fin de campagne - 1) Hydrographie.

1979. Dispersion de la pollution dans les pertuis charentais. Rapp. tech. gén. $79 \mathrm{p}$.

LEvinS, R. 1966. The strategy of model building in population biology. Am. Sci. 54(4) : 421-31.

LuTZ, R. A. 1980 . Mussel culture and harvest : a north american perspective Dev. Aquacult. Fish. Sci. 7.

MAJKowski, J. ET K. G. WAIwoOd. 1981. A procedure for evaluating the food biomass consumed by a fish population. Can. J. Fish. Aquat. Sci. 38 : 1199-1208

Nelder, J. A. ET R. MEAd. 1965. A simplex method for function minimization. Comput. J. $7: 308-313$.

Newell, R. C. 1979. Biology of intertidal animals. Marine Ecology Surveys LTD, Faversham, Kent. 773 p.

Peterson, C. H. ET R. BLACK. 1987. Resource depletion by active suspensionfeeders on tidal flats : influence of local density and tidal elevation. Limnol. Oceanogr. 32 : 143-166

Rodhouse, P. G. 1979. A note on the energy budget for an oyster population in a temperate estuary. J. Exp. Mar. Biol. Ecol. $37: 205-212$.

Rodhouse, P. G. ET M. O'Kelly. 1981. Flow requirements of the oysters Ostrea edulis L. and Crassostrea gigas Thunb. in an upwelling system of culture. Aquaculture $22: 1-10$.

SCHNUTE, J. 1982. A manual for easy non linear parameter estimation in fishery research with interactive microcomputer programs. Can. Tech. Rep. Fish. Aquat. Sci. $1140: 1-15$.

Urban, E. R. JR., G. D. Pruder et C. J. Langdon. 1983. Effect of ration on growth and growth efficiency of juveniles of Crassostrea virginica (Gmelin). J. Shellfish Res. 3(1): 51-57.

VERHAGEN, J. H. G. 1982. A distribution and population model of the mussel Mytilus edulis in Lake Grevelingen. 3rd International Conf. on State-ofthe-Art in Ecological Modelling. Colorado State University, May 24-28. 1982. 11 p. 